\title{
A Comparison Review based on Classifiers and Regression Models for the Investigation of Flash Floods
}

\author{
Talha Ahmed Khan ${ }^{1}$ \\ Usman Institute of Technology \\ Pakistan British Malaysian Institute \\ Universiti Kuala Lumpur \\ Malaysia \\ Muhammad Alam ${ }^{2}$ \\ Institute of Business Management, Pakistan \\ Universiti Kuala Lumpur \\ Malaysia
}

\author{
Kushsairy Kadir ${ }^{3}$ \\ British Malaysian Institute \\ Universiti Kuala Lumpur, Malaysia \\ Zeeshan Shahid ${ }^{4}$ \\ Institute of Business Management, Pakistan \\ M.S Mazliham 5 \\ Universiti Kuala Lumpur, Malaysia
}

\begin{abstract}
Several regions of the world have been affected by one of the natural disasters named as flash floods. Many villagers who live near stream or dam, they suffer a lot in terms of property, cattle and human lives loss. Conventional early warning systems are not up to the mark for the early warning announcements. Diversified approaches have been carried out for the identification of flash floods with less false alarm rate. Forecasting approaches includes some errors and ambiguity due to the incompetent processing algorithms and measurement readings. Process variables like stream flow, water level, water color, precipitation velocity, wind speed, wave's pattern and cloud to ground (CG) flashes have been measured for the robust identification of flash floods. A vibrant competent algorithm would be required for the investigation of flash floods with less false alarm rate. In this research paper classifiers have been applied on the collected data set so that any researcher could easily know that which classifier is competent and can be further enhanced by combining it with other algorithms. A novel comprehensive parametric comparison has been performed to investigate the classification accuracy for the robust classification of false alarms. For the better accuracy more than one process variables have been measured but still contained some false alarm. Appropriate combination of sensor was integrated to increase the accuracy in results as multi-modal sensing device has been designed to collect the data. Linear discriminant analysis, logistic regression, quadratic support vector machine, $\mathrm{k}$ nearest neighbor and Ensemble bagged tree have been applied to the collected data set for the data classification. Results have been obtained in the MATLAB and discussed in detail in the research paper. The worst accuracy of the classification (62\%) has been achieved by the coarse $\mathrm{k}$-NN classifier that means coarse k-NN produced $38 \%$ false negative rate that is not acceptable in the case of forecasting. Ensemble bagged trees produced best classification results as it achieved $99 \%$ accuracy and $1 \%$ error rate. Furthermore, according to the comprehensive parametric comparison of regression models Quadratic SVM found to be the worst with mean square error of 0.5551 and time elapsed 13.159 seconds. On the other hand, Exponential Gaussian process regression performed better than
\end{abstract}

the other existing approaches with the minimum root mean squared error of $\mathbf{0 . 0 0 0 2}$ and prediction speed of 35000 observations per second.

Keywords-Flash floods; classification; SVM; $k$-NN; logistic regression; quadratic SVN; ensemble bagged trees; exponential GPR

\section{INTRODUCTION}

Low cost effective solution has been designed using Android phone and Arduino. Echolocation strategy has been applied to measure the water level [1]. Bunch of sensors were deployed from the upper stream to the village. Sensors nodes were designed to observe the data. Supervisor control data acquisition (SCADA) based system was designed to forecast the floods on real time basis [2]. Torrential downpour can be considered as the main cause of flash floods. Heavy precipitation for the short time known as torrential downpour. Two meteorological radars have been used to observe the torrential downpour in Czech Republic [3]. Ultrasonic sensor based observations have been analyzed to determine the dam level [4]. Data from 2007 to 2010 were collected from three stations of Selangor to design a novel model for the prediction of flash floods using ANN. Feed forward back propagation with the tangent sigmoid function was proposed to estimate the floods. The process variables like humidity, rainfall, and temperature were taken as input and the rainfall data from the stations were set as the targets. The proposed model best results [4]. Another research elaborated a novel method by measuring the magnetic field lines by tesla meter or magnetometer to predict the flash floods. Research proved by showing the results that during the flash floods magnetic field line reduced abruptly therefore magnetic field lines that are radiated from the center of the earth can be regarded as the significant yardstick to measure the flash floods [5]. A practical early warning system must detect the flash floods in appropriate timings as there is no use of the system if the flood 
is detected after the starting of the event. Keeping in the mind the time constraint a flood prediction model using hybrid approach NNARX (Neural Network Autoregressive with Exogenous Input) with EKF (extended Kalman Filter) was developed. 120 samples of the data set were tested and results showed that hybrid approach worked better [6]. In a previous method a novel solution was suggested by measuring the carbon dioxide levels in the environment and soil flux as the newly discovered phenomena proved that plants take less water due to the increased amount of carbon dioxide levels. Multi-layer perceptron was applied on the collected data set to reduce the false alarms in determining the flash floods [7]. IT based flash flood monitoring was performed for the immediate emergency rescue of the affected people in Jeddah [8]. Majority casualties happen due to the lack of the data and information regarding the propagation of the flash flood. Therefore, an urban flash flood monitoring was performed to know the actual and predicted flow of the flash floods for the evacuation announcement [9]. Kalman filtering, fuzzy logic, clustering, Neural network autoregressive model with exogenous input (NNARX), Particle swarm optimization (PSO) and Support vector machine have been applied for the prediction and estimation of flash floods [10]. Fuzzy logic based a disaster management device has been designed for the announcement of exit routes during the hazard [11]. Ensemble learning model has also been designed for the better generalization model of classification [12]. The false positive rate $(\mathrm{FP})$ is the date values which have been estimated wrong due to the presence of error. The negative false rate $(\mathrm{FN})$ is the data that is defined falsely as negative [13]. False alarm ratio relies on the relation of the complexity and anisotropy of the sea-floor Williams et al. [14] [15]. Gaussian process regression model can be acknowledged as the competent approach for solving non-linear regression issues. It performs regression in a simple way parameterization and Bayesian. It also removes ambiguity and uncertainty in the prediction of event [16]. Data driven approaches are usually capable to determine the complex and non-linear data to be transformed for the prediction of the event [17]. Support vector machine was developed in 1990s and became popular among the classification model to its better learning generalization [18]. Signal attenuation and distortion in Television satellites due to the rainfall was observed and flooding was mapped. It has been analyzed that $\mathrm{Ku}$ band frequencies varied due to rain fall and climate change. Simulated maps of flash floods were compared with the existing mapping methods to validate the approach [22].

Table I demonstrates the tabular chart for the comparison of AI based algorithms. Root mean square error has been a yardstick to estimate the performance of the algorithms. The results of actual run time data using existing approach of MLP-PSO show probability of $95.15 \%$. The proposed algorithm of ANN-PSO has performed the investigation of flash flood with 0.0047 error probability and enhanced accuracy [20].
TABLE. I. COMPARATIVE ANALYSIS OF VARIOUS APPROACHED FOR THE FLASH FLOOD INVESTIGATION [20]

\begin{tabular}{|l|l|l|l|l|l|}
\hline $\begin{array}{l}\text { Performan } \\
\text { ce Indices }\end{array}$ & ANN & SVM & ANFIS & NNARX & $\begin{array}{l}\text { ANN- } \\
\text { PSO }\end{array}$ \\
\hline RMSE & 0.194 & 0.390 & 0.116 & 0.090 & 0.0047 \\
\hline Best Fit & 73 & 64 & 78 & 80.10 & 98.7 \\
\hline Results & $\begin{array}{l}\text { Satisfacto } \\
\text { ry }\end{array}$ & $\begin{array}{l}\text { Unsatisfacto } \\
\text { ry }\end{array}$ & $\begin{array}{l}\text { Satisfacto } \\
\text { ry }\end{array}$ & $\begin{array}{l}\text { Satisfacto } \\
\text { ry }\end{array}$ & $\begin{array}{l}\text { Satisfacto } \\
\text { ry }\end{array}$ \\
\hline $\begin{array}{l}\text { Hourly } \\
\text { Data }\end{array}$ & $6 \mathrm{hrs}$ & $6 \mathrm{hrs}$ & $3 \mathrm{hrs}$ & $3 \mathrm{hrs}$ & $3 \mathrm{hrs}$ \\
\hline Accuracy & 73 & 64 & 78 & 80.16 & 98.99 \\
\hline Precision & Medium & Low & High & High & High \\
\hline Reliability & Medium & Low & High & High & High \\
\hline $\begin{array}{l}\text { Power } \\
\text { Utilixation }\end{array}$ & Limited & Limited & Limited & Limited & Limited \\
\hline
\end{tabular}

\section{Problem Statement}

It has been highlighted in the literature review that sensors and transducers produce false alarms. Errors are generated usually in instrumentation and measurement [10]. Sometime prediction of flash floods can be wrong due to the incompetent decision algorithms and poor sensitivity of sensors. Due to the increased number of false alarms a competent and vigorous classifier and regression model was required for the discrimination of true positive vale and false positive value. Sensors data values may contain false alarms and missed values [21].

\section{MATERIALS AND MethodS}

Multi-modal sensing device was developed to collect the data from any sea shore. The data has been collected from the sea shore of Kund Malir, Pakistan. Selection of transducers was not an easy task as appropriate combination of competent sensors was needed. According to the literature review almost all the parameters have been used for the flash floods investigation.

\section{A. Multi-Resolution Sensing Device for Data Collection}

Fig. 1 shows that a device has been developed for the investigation of flash flood as a hazard monitoring device. Multi-modal sensing device comprised of the following sensors: (a) pressure (b) temperature (c) water level (d) gas sensor for detecting $\mathrm{CO}_{2}$ and (e) ultrasonic sensor. Selection of sensors was very complex task as bunch of appropriate sensors must be used for the accurate and precise results without any false alarm rate [21].

\section{B. Fundamental Flow Diagram}

In Fig. 2, Ultrasonic sensor, Passive infrared sensor, MQ2 sensor, humidity sensor, pressure and temperature sensors have been used to measure the data near the sea shore of Kund Malir, Pakistan. Data labeling was performed. The data may contain random, missed and repetitive values it must be filtered or normalized before the processing. Therefore, robust classification and regression model were needed. 


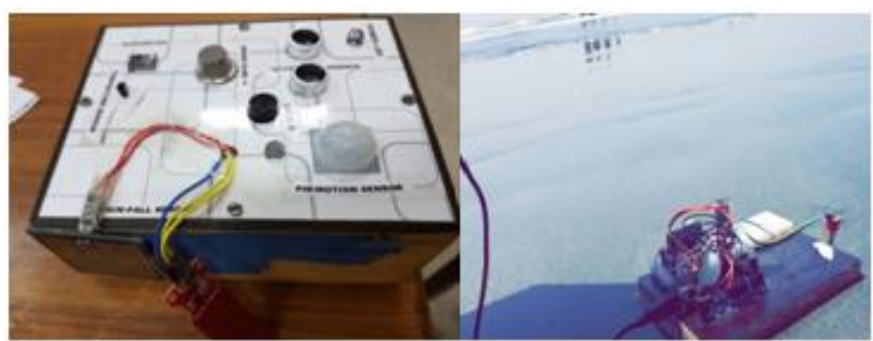

Fig. 1. Multi-Resolution Data Collection Device.

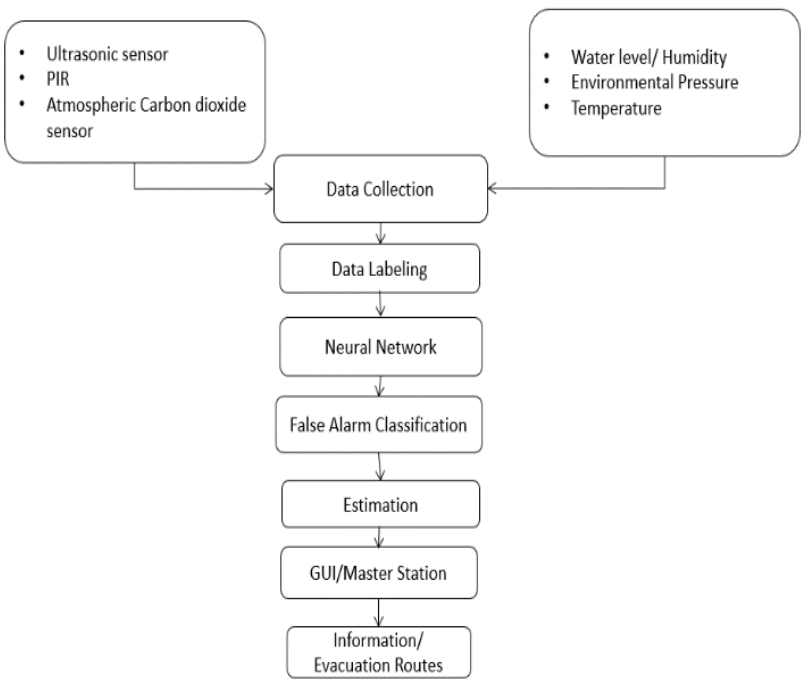

Fig. 2. Main Flow Diagram.

\section{Collected Data Set}

Table II shows that the data set has been collected and all the values have been saved in parallel at the same time. All the sensors have been correlated to each other. More than ten thousand number of instances were recorded and observed for the data processing having total six attributes. Six attributes are the different sensor data values that have been represented in the Table I.

\section{Threshold}

Table III represents the threshold and range related to the sensor values. All of the sensors must exceed the threshold in order to activate the trigger function. Moreover, all the sensors have been interfaced to each other. Sensors produced random results due to the high wind speed. Change of venue for data collection requires modification in threshold of sensors depending on the meteorology of location.

TABLE. II. Collected Data Set From the Multi-Modal Sensing DEVICE [21]

\begin{tabular}{|l|l|l|l|l|l|}
\hline PIR & $\begin{array}{l}\text { Distance } \\
(\mathrm{mm})\end{array}$ & Rainfall & $\begin{array}{l}\mathrm{CO}_{2} \\
(\mathrm{ppm})\end{array}$ & $\begin{array}{l}\text { Temperature } \\
\left({ }^{\circ} \mathrm{C}\right)\end{array}$ & $\begin{array}{l}\text { Pressure } \\
(\text { hectopascal })\end{array}$ \\
\hline 0 & 300 & 0 & 0 & 30.13 & 100270.6 \\
\hline 0 & 37.42 & 458 & 539 & 29.95 & 100268.4 \\
\hline 0 & 37.18 & 459 & 533 & 29.71 & 100277.5 \\
\hline 0 & 3661.34 & 453 & 418 & 29.6 & 100279.8 \\
\hline 0 & 3654.66 & 453 & 397 & 29.46 & 100277.5 \\
\hline 0 & 3691.18 & 450 & 356 & 37 & 100280.1 \\
\hline 0 & 3691.18 & 450 & 356 & 29.4 & 100280.1 \\
\hline
\end{tabular}

\begin{tabular}{|c|c|c|c|c|c|}
\hline 1 & 4.51 & 504 & 355 & 28 & 110424.2 \\
\hline 1 & 4.39 & 494 & 412 & 23 & 110424.2 \\
\hline 1 & 4.96 & 485 & 399 & 23 & 110424.2 \\
\hline 1 & 4.84 & 479 & 382 & 23 & 100283.5 \\
\hline 1 & 5.06 & 476 & 349 & 23 & 110424.2 \\
\hline 1 & 4.66 & 483 & 400 & 23 & 110424.2 \\
\hline 1 & 4.66 & 478 & 387 & 23 & 110424.2 \\
\hline 1 & 32.45 & 551 & 392 & 23 & 110424.2 \\
\hline 0 & 22.45 & 512 & 387 & 23 & 110424.2 \\
\hline 0 & 29.64 & 518 & 388 & 23 & 110424.2 \\
\hline 1 & 3619.3 & 529 & 389 & 23 & 110424.2 \\
\hline 1 & 78.39 & 498 & 386 & 23 & 110424.2 \\
\hline 0 & 0 & 491 & 385 & 23 & 110424.2 \\
\hline 0 & 30.13 & 517 & 397 & 23 & 110424.2 \\
\hline 0 & 36.96 & 501 & 395 & 23 & 110424.2 \\
\hline 0 & 31.92 & 496 & 392 & 23 & 110424.2 \\
\hline 0 & 32.95 & 497 & 390 & 23 & 110424.2 \\
\hline 0 & 41.18 & 503 & 387 & 23 & 110424.2 \\
\hline 0 & 135.14 & 494 & 385 & 23 & 110424.2 \\
\hline 0 & 3846.76 & 496 & 385 & 23 & 110424.2 \\
\hline 0 & 6.47 & 519 & 393 & 56 & 110424.2 \\
\hline 0 & 104.96 & 506 & 389 & 23 & 110424.2 \\
\hline 0 & 103.76 & 498 & 387 & 23 & 110424.2 \\
\hline 0 & 31.2 & 488 & 385 & 25 & 110424.2 \\
\hline 0 & 104.63 & 486 & 386 & 25 & 110424.2 \\
\hline 0 & 34.51 & 535 & 389 & 25 & 110424.2 \\
\hline 1 & 6.72 & 530 & 1014 & 25 & 110424.2 \\
\hline 1 & 20.91 & 527 & 1012 & 25 & 110424.2 \\
\hline 0 & 101.85 & 511 & 1010 & 25 & 110424.2 \\
\hline 0 & 3749.04 & 499 & 347 & 25 & 110424.2 \\
\hline 0 & 36.96 & 493 & 303 & 25 & 110424.2 \\
\hline 0 & 9.3 & 492 & 324 & 25 & 110424.2 \\
\hline 0 & 0 & 493 & 388 & 25 & 110424.2 \\
\hline 0 & 110.74 & 492 & 363 & 25 & 110424.2 \\
\hline 0 & 105.54 & 495 & 341 & 25 & 110424.2 \\
\hline 0 & 23.86 & 496 & 392 & 25 & 110424.2 \\
\hline 0 & 99.76 & 498 & 1012 & 25 & 110424.2 \\
\hline 0 & 3279.56 & 502 & 1012 & 25 & 110424.2 \\
\hline 0 & 75.99 & 510 & 1012 & 25 & 110424.2 \\
\hline 0 & 3259.63 & 536 & 1010 & 25 & 110424.2 \\
\hline 0 & 37.8 & 534 & 1010 & 25 & 110424.2 \\
\hline 0 & 3279.9 & 522 & 1010 & 25 & 110424.2 \\
\hline 0 & 35.02 & 517 & 1011 & 25 & 110424.2 \\
\hline 0 & 3223.39 & 516 & 1011 & 25 & 110424.2 \\
\hline 0 & 30.96 & 543 & 1010 & 25 & 110424.2 \\
\hline 0 & 3244.81 & 557 & 1009 & 25 & 110424.2 \\
\hline 0 & 32.29 & 571 & 1009 & 25 & 110424.2 \\
\hline 0 & 3233.67 & 553 & 1010 & 25 & 110424.2 \\
\hline 0 & 35.19 & 530 & 1010 & 23 & 110424.2 \\
\hline 0 & 35.79 & 521 & 1010 & 23 & 110424.2 \\
\hline 0 & 3300.48 & 522 & 1010 & 23 & 110424.2 \\
\hline 0 & 29.72 & 549 & 1009 & 23 & 110424.2 \\
\hline
\end{tabular}

TABLE. III. THRESHOLD VALUES FOR SENSORS

\begin{tabular}{|l|l|l|}
\hline & $\begin{array}{l}\text { MINIMUM } \\
\text { LIMIT }\end{array}$ & $\begin{array}{l}\text { MAXIMUM } \\
\text { LIMIT }\end{array}$ \\
\hline PIR & 1 & \\
\hline DISTANCE & 0 & 50 \\
\hline RAINFALL & $>300$ \\
\hline CO2 & $>600$ \\
\hline TEMPERATURE & 0 & 50 \\
\hline PRESSURE & $>5000$ \\
\hline ALTIMETER & $>1000$ \\
\hline
\end{tabular}




\section{EXTENSIVE PARAMETRIC COMPARISON OF DATA CLASSIFIERS}

Table IV demonstrated that Comprehensive parametric comparison has been performed to investigate the classification accuracy for the robust classification of false alarm in predicting flash floods. Linear discriminant analysis, logistic regression, quadratic support vector machine, knearest neighbor and Ensemble bagged tree have been applied to the collected data set for the data classification. Initially seventy-five percent of the data were used as a training and other 25 percent data was saved for the testing purpose. Both of the data files training and testing were converted into the variable so that it may utilized in the MATLAB as all the simulations have been performed in the MATLAB. MATLAB based simulations produced the confusion matrix and all the parametric results which have been presented in the table. The worst accuracy of the classification $(62 \%)$ has been achieved by the coarse k-NN classifier that means coarse k-NN produced $38 \%$ false negative rate that is not acceptable in the case of forecasting. Ensemble bagged trees produced best classification results as it achieved $99 \%$ accuracy and $1 \%$ error rate.

\section{A. Linear Discriminant Analysis}

Fig. 3 explains that data set was trained for the linear discriminant classification and it produced confusion matrix and other results. Confusion matrix showed that LDA achieved $89 \%$ true positive rate and 11 false negative rate with $97 \%$ accuracy. Prediction speed and training time was found to be 12000 observation/seconds and 1.5238 seconds respectively. This classification model was up to the mark but accuracy can be further improved. Data can be regularized in discriminant analysis classifier for the robust classification model.

\section{B. Logistic Regression Classification Model}

Fig. 4 represents that Logistic regression classification model was developed in the MATLAB using the collected data set. The classification model achieved $96.4 \%$ accuracy. Prediction time and training time was found to be 15000 observation/second and 3.9633 seconds. It took almost double time to classify the faulty data compared to the linear discriminant analysis with slight less accuracy.

\section{Quadratic Support Vector Machine Classification Model}

Fig. 5 illustrates that Quadratic support vector machine classifier has been applied to the collected data set form the sea shore of Kund Malir. The model is based on predict SVM model in which predictors have been defined in the matrix and then comparison would be performed between the observed and predicted. The trained Quadratic SVM model may be compact or full. The trained model has been exported for the testing purpose. yout $=$ predict $(Q S V M M o d e l, X)$. Quadratic support vector machine classifier model achieved $93 \%$ true positive rate and $7 \%$ False negative rate in the confusion matrix. $96.8 \%$ accuracy achieved by Q-SVM in 0.9237 seconds of training time. Prediction speed was found to be 40000 observations per second.
TABLE. IV. PARAMETRIC COMPARISON OF LDA, LR, QSVM, K-NN AND ENSEMBLE

\begin{tabular}{|l|l|l|l|l|l|}
\hline $\begin{array}{l}\text { Classification } \\
\text { Models }\end{array}$ & $\begin{array}{l}\text { True } \\
\text { Positive } \\
\text { rate } \\
(\%)\end{array}$ & $\begin{array}{l}\text { False } \\
\text { Negative } \\
\text { rate }(\%)\end{array}$ & $\begin{array}{l}\text { Accuracy } \\
(\%)\end{array}$ & $\begin{array}{l}\text { Prediction } \\
\text { speed per } \\
\text { second }\end{array}$ & $\begin{array}{l}\text { Training } \\
\text { Time (s) }\end{array}$ \\
\hline $\begin{array}{l}\text { Linear } \\
\text { Discriminant }\end{array}$ & 89 & 11 & 97.0 & 12000 & 1.5238 \\
\hline $\begin{array}{l}\text { Logistic } \\
\text { Regression }\end{array}$ & 93 & 7 & 96.4 & 15000 & 3.9633 \\
\hline $\begin{array}{l}\text { Quadratic } \\
\text { SVM }\end{array}$ & 93 & 7 & 96.8 & 40000 & 0.9327 \\
\hline Fine k-NN & 96 & 4 & 98.6 & 9600 & 1.5307 \\
\hline $\begin{array}{l}\text { Medium k- } \\
\text { NN }\end{array}$ & 91 & 9 & 96.6 & 19000 & 0.927 \\
\hline Coarse k-NN & 62 & 38 & 89.3 & 16000 & 0.90243 \\
\hline $\begin{array}{l}\text { Ensemble } \\
\text { Bagged Trees }\end{array}$ & 99 & 1 & 99.4 & 2800 & 6.4101 \\
\hline
\end{tabular}

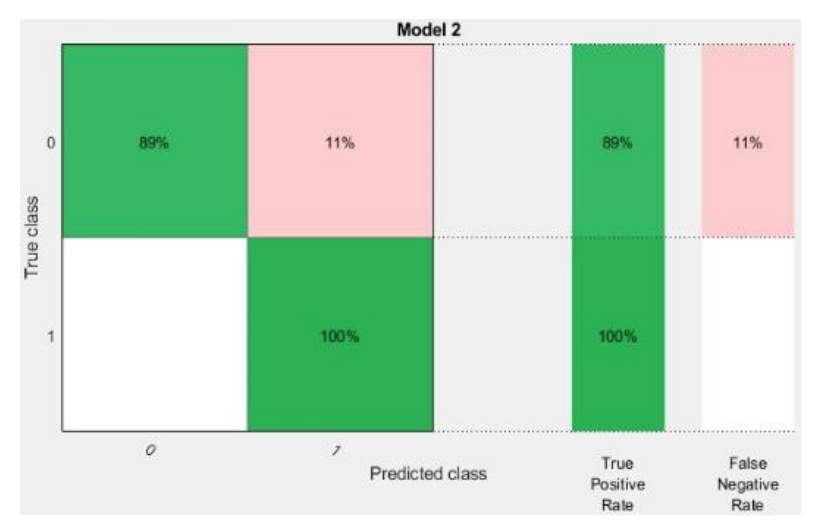

Fig. 3. Linear Discriminant Analysis Confusion Matrix.

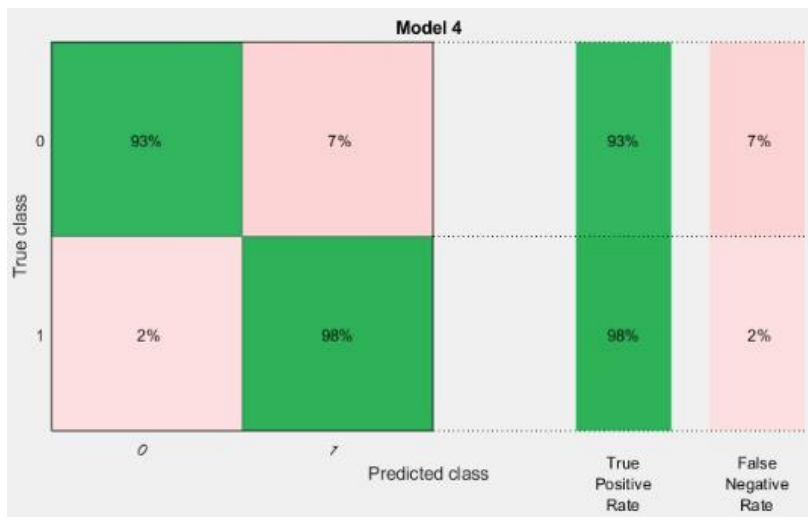

Fig. 4. Logistic Regression Confusion Matrix.

\section{Fine k-Nearest Neighbor Classification Model}

Fig. 6 shows the confusion matrix for the Fine k-nearest neighbor algorithm. Fine k-nearest neighbor classification model has been developed by measuring the standardized Euclidian distance.

$$
E=\left(u_{u}-V_{v}\right) B^{-1}\left(u_{u}-V_{v}\right)
$$




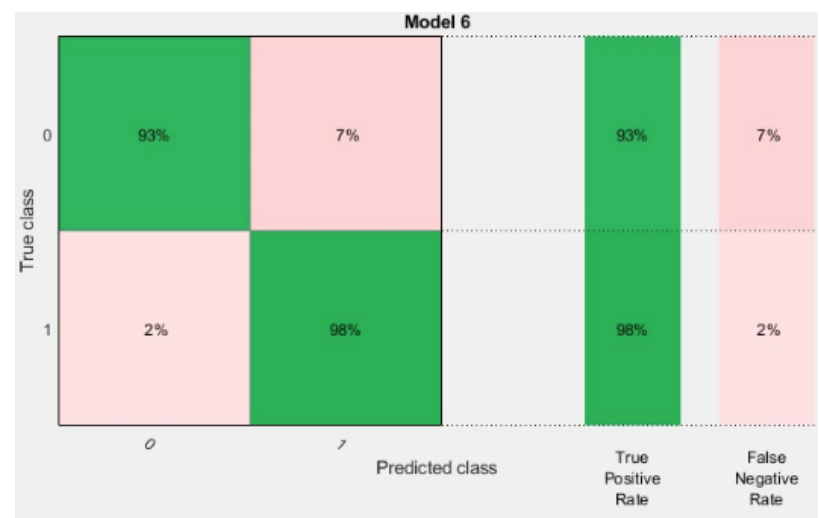

Fig. 5. Q-SVM Confusion Matrix.

Where, $\mathrm{U}$ and $\mathrm{V}$ are the data matrix. $\mathrm{B}$ can be considered as the diagonal matrix and $\mathrm{S}$ can be acknowledged as scaling factor. Model was trained in the MATLAB using collected data set. $96 \%$ true positive rate and $4 \%$ of false negative rate was achieved in this classification model. The fine k-NN classification model achieved $98.6 \%$ accuracy with training time of 1.5307 second and prediction speed of 9600 observations per second.

\section{E. Medium k-Nearest Neighbor Classification Model}

Fig. 7 explains that medium k-NN classifiers have been used widely as a bench mark for learning. k-NN classifier has the capability to be modified easily as well. $\mathrm{X}$ can be considered as the numeric data for the training. Moreover, Chebychev distance, Euclidean distance, city block distance and Minkwoski distance can be measured for determining the $k d$-Tree. Medium k-nearest neighbor classification model produced $19 \%$ true positive rate and $9 \%$ false negative rate. $96.6 \%$ accuracy was achieved with training time of 0.927 second and 19000 observations per second.

\section{F. Coarse k-Nearest Neighbor Classification Model}

In Fig. 8, the worst accuracy of the classification (62\%) has been achieved by the coarse k-NN classifier that means coarse $\mathrm{k}-\mathrm{NN}$ produced $38 \%$ false negative rate that is not acceptable in the case of forecasting.

\section{G. Ensemble Bagged Trees Classification Model}

Fig. 9 represented the confusion matrix of Ensemble Bagged Trees. The Ensemble bagged trees has been applied to the data set for the better decision. The motive of the ensemble is to construct a better learning model which can produce better classification performance on the applied data set. Yfit $=$ ensemblebaggedtree $(B, X)$ returns a vector of forecasted responses for the predictor data in the table or matrix $X$, based on the ensemble of bagged decision trees B. Yfit is a cell array of character vectors for classification and a numeric array for regression. By default, predict takes a democratic (non-weighted) average vote from all trees in the ensemble. $\mathrm{X}$ can be considered as a numeric Matrix. Ensemble Bagged Tree learning model for classification achieved best performance accuracy of $99.4 \%$ with prediction speed of 2800 observations/second and training time is 6.4101 seconds. $99 \%$ True positive rate and
$1 \%$ false negative rate was achieved by the Ensemble Bagged Trees.

\section{H. Graphical Illustration of Model}

Fig. 10 displays the Graphical analysis of ensemble bagged tree prediction model has been represented in figure no. 9. Blue color shows the output value of "zero" and orange color represents "one". It can be easily observed from the results that Ensemble bagged tree performed better than the other existing classifiers.

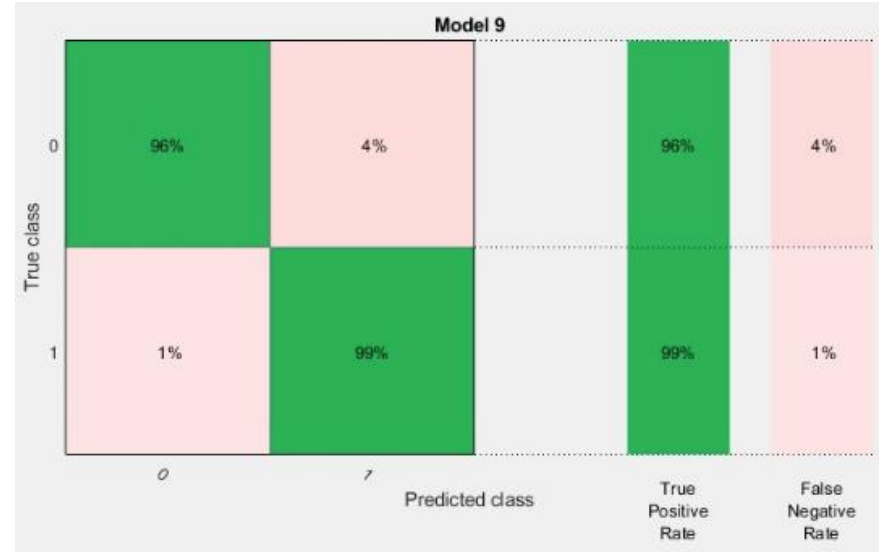

Fig. 6. Fine k-Nearest Neighbor Confusion Matrix.

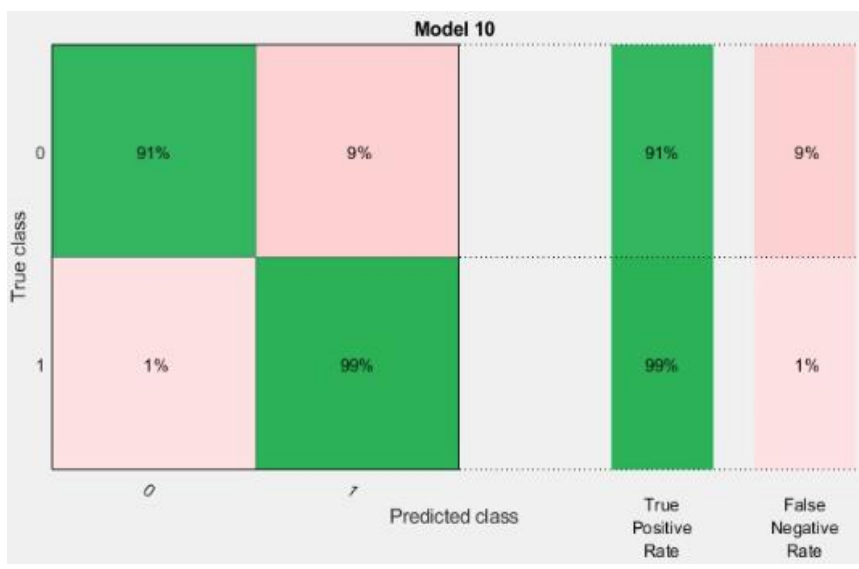

Fig. 7. Medium k-NN Confusion Matrix.

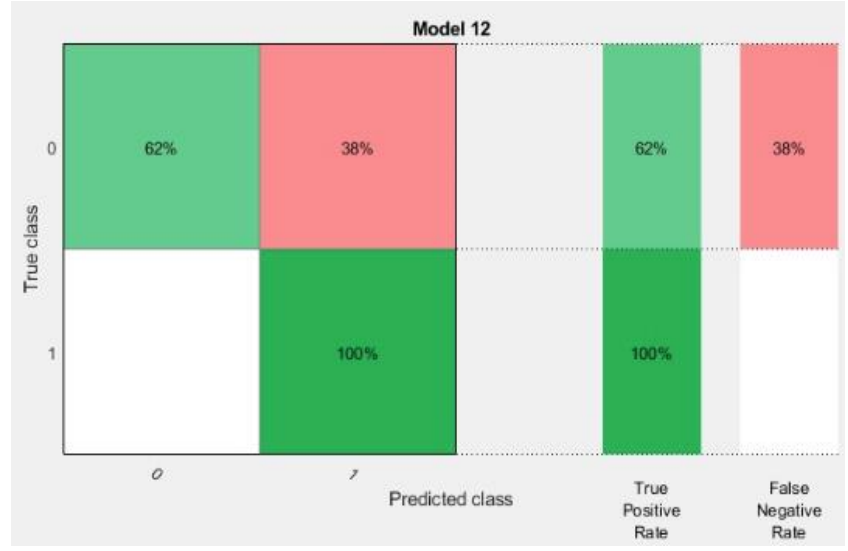

Fig. 8. Coarse k-NN Confusion Matrix. 


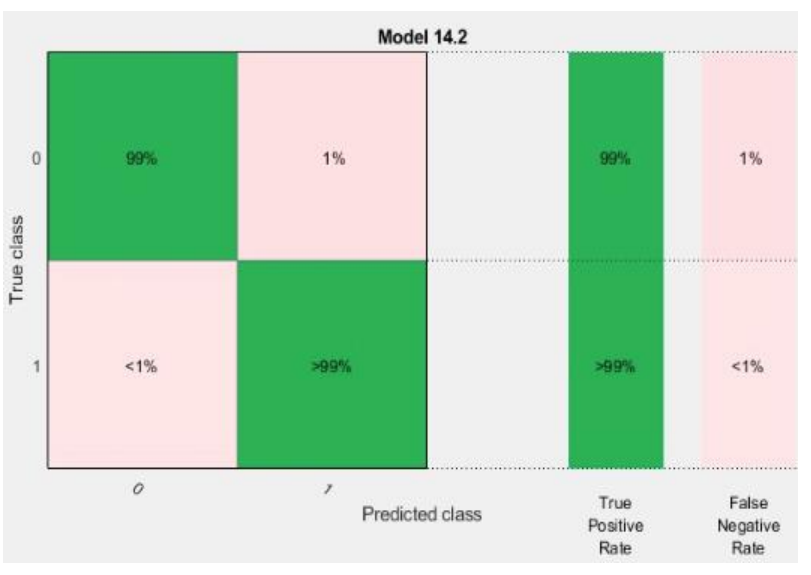

Fig. 9. Ensemble Bagged Trees Confusion Matrix.

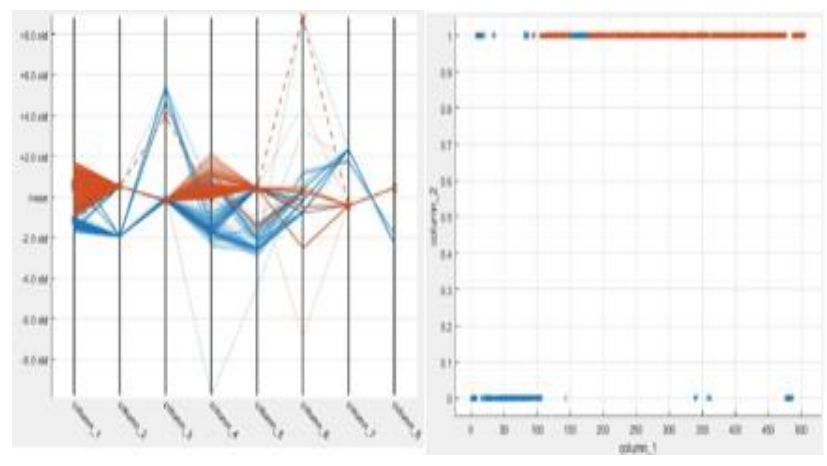

Fig. 10. Graphical Analysis of Prediction Model.

\section{EXHAUSTIVE PARAMETRIC COMPARISON OF REGRESSION MODELS}

Table V shows the comprehensive parametric comparison for finding out the best performer regression model. LR, interactions linear, robust linear, step wise linear, linear support vector machine, Quadratic SVM, Gaussian SVM, Rational quadratic GPR, Exponential GPR and ensemble bagged trees regression models have been developed for the prediction of flash floods. These regression models have been applied on the collected data set. According to the comprehensive parametric comparison of regression models Quadratic SVM found to be the worst with mean square error of 0.5551 and time elapsed 13.159 seconds. On the other hand, Exponential Gaussian process regression performed better than the other existing approaches with the minimum root mean squared error of 0.0002 and prediction speed of 35000 observations per second.

\section{A. Graphical Illustration of Linear Regression, Interactions Linear, Robust Linear and Step Wise linear}

Fig. 11 demonstrates the graphical illustration of model 1 (Linear Regression), Model 2 (Interactions Linear), Model 3 (Robust Linear) and Model 4 (step wise linear). Moreover, Blue color represents the true data and yellow color depicts the predicted data. The graphs have been plotted between number of records and flood response (hurricane response).
TABLE. V. PARAMETRIC COMPARISON OF REGRESSION APPROACHES

\begin{tabular}{|l|l|l|l|l|l|l|}
\hline $\begin{array}{l}\text { Predictive } \\
\text { Models }\end{array}$ & RMSE & $\mathrm{R}^{2}$ & $\mathrm{MSE}$ & $\mathrm{MAE}$ & $\begin{array}{l}\text { Prediction } \\
\text { speed per } \\
\text { second }\end{array}$ & $\begin{array}{l}\text { Training } \\
\text { Time (s) }\end{array}$ \\
\hline $\begin{array}{l}\text { Linear } \\
\text { Regression }\end{array}$ & 0.1900 & 0.82 & 0.0361 & 0.113 & 11000 & 4.5756 \\
\hline $\begin{array}{l}\text { Interactions } \\
\text { Linear }\end{array}$ & 0.1087 & 0.94 & 0.0118 & 0.044 & 39000 & 0.8332 \\
\hline $\begin{array}{l}\text { Robust } \\
\text { Linear }\end{array}$ & 0.267 & 0.65 & 0.0712 & 0.071 & 80000 & 0.898 \\
\hline $\begin{array}{l}\text { Step Wise } \\
\text { Linear }\end{array}$ & 0.1118 & 0.94 & 0.0125 & 0.046 & 70000 & 3.779 \\
\hline $\begin{array}{l}\text { Linear } \\
\text { SVM }\end{array}$ & 0.2316 & 0.73 & 0.0536 & 0.127 & 47000 & 11 \\
\hline $\begin{array}{l}\text { Quadratic } \\
\text { SVM }\end{array}$ & 0.5551 & -0.53 & 0.0308 & 0.322 & 73000 & 13.159 \\
\hline $\begin{array}{l}\text { Gaussian } \\
\text { SVM }\end{array}$ & 0.0721 & 0.97 & 0.0052 & 0.045 & 140000 & 0.818 \\
\hline $\begin{array}{l}\text { Rational } \\
\text { Quadratic } \\
\text { GPR }\end{array}$ & 0.0006 & 1 & $3 \times 10^{-7}$ & $9 \times 10^{-5}$ & 22000 & 1.1555 \\
\hline $\begin{array}{l}\text { Exponential } \\
\text { GPR }\end{array}$ & 0.0002 & 1 & $4 \times 10^{-8}$ & $3 \times 10^{-5}$ & 35000 & 2.1752 \\
\hline $\begin{array}{l}\text { Ensemble } \\
\text { Bagged } \\
\text { Trees }\end{array}$ & 0.0750 & 0.97 & 0.0056 & 0.0222 & 34000 & 2.3892 \\
\hline
\end{tabular}

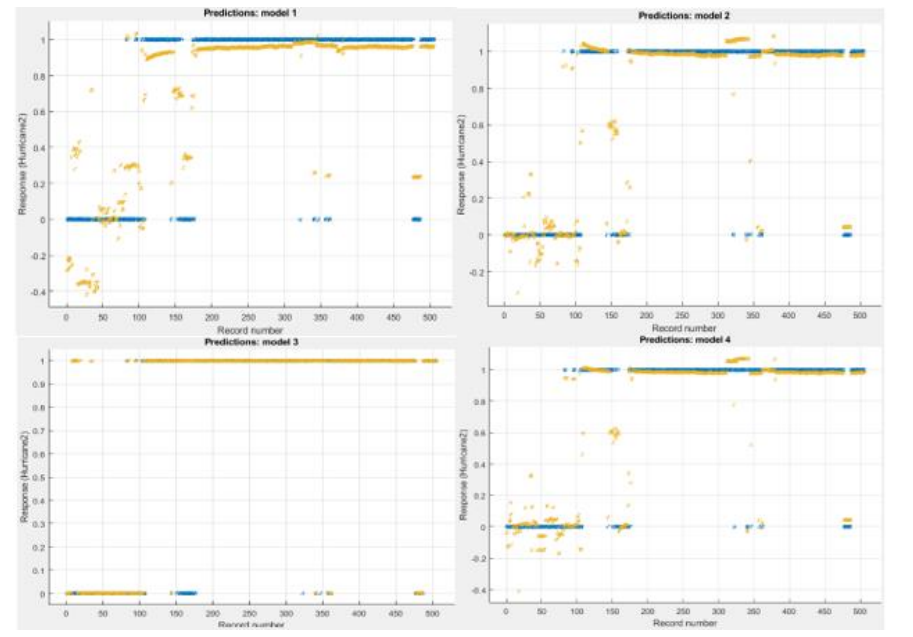

Fig. 11. Graphical Illustration of Prediction Model 1, Model 2, Model 3, Model 4.

\section{B. Graphical Illustration of Linear SVM, Quadratic SVM, Gaussian SVM and Rational Quadratic GPR}

Fig. 12 demonstrated the graphical illustration of model 5 (Linear SVM), Model 6 (Quadratic SVM), Model 7 (Gaussian SVM) and Model 8 (Quadratic GPR). Moreover, Blue color represents the true data and yellow color depicts the predicted data. The graphs have been plotted between number of records and flood response (hurricane response). 


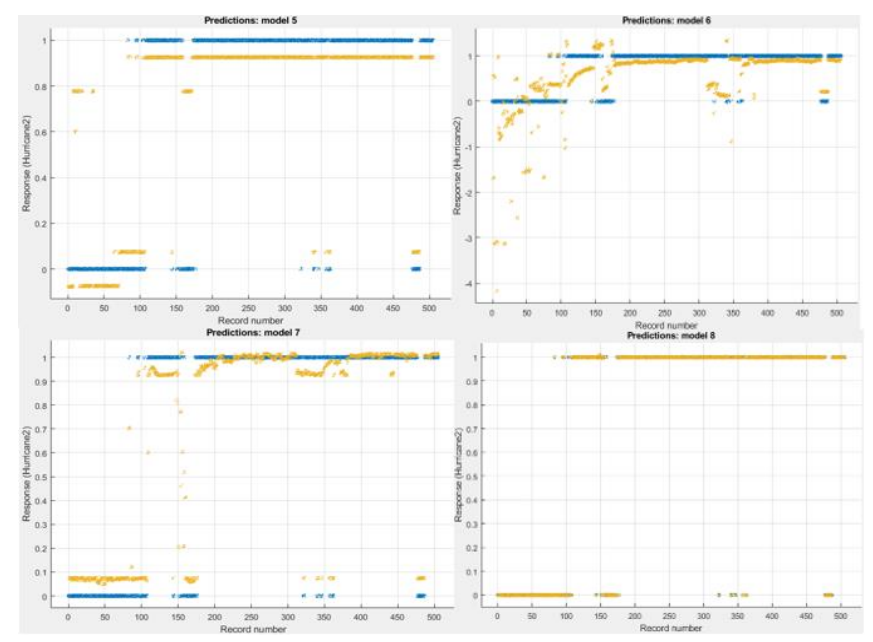

Fig. 12. Graphical Illustration of Prediction Model 5, Model 6, Model 7, Model 8.

\section{Graphical Illustration of Exponential GPR and Ensembled Bagged Trees}

Fig. 13 portrayed the graphical analysis of model 9 (Exponential GPR) and model 10 (Ensemble Bagged Trees). Moreover, Blue color represents the true data and yellow color depicts the predicted data. The graphs have been plotted between number of records and flood response (hurricane response). (Gaussian Processes). For set $\mathrm{S}$, mean function $\mu$ : $\mathrm{S} 7 \rightarrow \mathrm{R}$ and any covariance function (also called kernel) $\mathrm{k}$ : $\mathrm{S} \times \mathrm{S} 7 \rightarrow \mathrm{R}$, there exists a GP $\mathrm{f}(\mathrm{x})$ on $\mathrm{S}$,

s.t. $E[f(x)]=\mu(x), \operatorname{Cov}(f(x s), f(x t))=k(x s, x t), x, x s, x t \in S$

It denotes $\mathrm{f} \sim \mathrm{GP}(\mu, \mathrm{k})$. For a regression problem $\mathrm{y}=\mathrm{f}(\mathrm{x})$ $+\varepsilon$, by Gaussian process method the unknown function $f$ is assumed to follow a $\operatorname{GP}(\mu, \mathrm{k})$. Given $\mathrm{n}$ pairs of observations $(\mathrm{x} 1, \mathrm{y} 1), \ldots,(\mathrm{xn}, \mathrm{yn})$,

we have

$y=f(X)+\varepsilon$

where $y=[y 1, y 2, \ldots, y n] T$ are the outputs, $X=[x 1, x 2$, $\ldots, \mathrm{xn}] \mathrm{T}$ are the inputs, and $\varepsilon=[\varepsilon 1, \varepsilon 2, \ldots, \varepsilon n] \mathrm{T}$ are independent identically distributed Gaussian noise with mean 0 and variance $\sigma 2 \mathrm{n}[19]$.

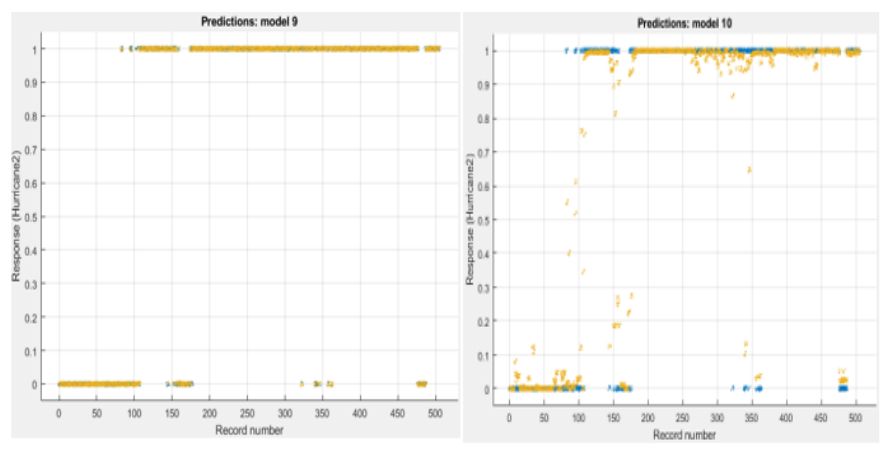

Fig. 13. Graphical Analysis of Prediction Model 9 and Model 10.

\section{CONCLUSION AND FUTURE WORK}

In this research paper various classification model and regression model have been investigated to determine the competent approach for the identification of flash floods with less false alarm rates. Parametric comparison has been shown so that researchers may have an idea about the technical and practical implications related to the flash flood research. Actually the collected data or observed data may contain inconsistent, missed or random values. Therefore, vigorous classification and predictive model is required for the accurate and precise identification of the flash floods. Moreover, Bayesian regularization and scaled conjugate gradient were applied to the data set. Results showed that scaled conjugate gradient performed better. Comprehensive parametric comparison of classifiers and regression models has been performed and compare to find out the better classification and regression model. It can be concluded that the worst accuracy of the classification (62\%) has been achieved by the coarse $\mathrm{k}-\mathrm{NN}$ classifier that means coarse $\mathrm{k}-\mathrm{NN}$ produced $38 \%$ false negative rate that is not acceptable in the case of forecasting. Ensemble bagged trees produced best classification results as it achieved $99 \%$ accuracy and 1\% error rate. According to the comprehensive parametric comparison of regression models Quadratic SVM found to be the worst with mean square error of 0.5551 and time elapsed 13.159 seconds. On the other hand, Exponential Gaussian process regression performed better than the other existing approaches with the minimum root mean squared error of 0.0002 and prediction speed of 35000 observations per second.

\section{ACKNOWLEDGMENT}

Prof. Dr. Mohammad Alam's (Universiti Kuala Lumpur, Institute of Business Managament) has suggested me how to develop multi-modal sensing gadget for the collection of data. Authors are extremely thankful to Dr. Muhammad Alam as he recommended some competent classifier algorithms as his expertise are in Artificial Intelligence. Dr. Kushsairy Kadir (UniKL-BMI) and Dr. Zeeshan Shahid (IoBM) were found to be true mentor as they always mentored and improved proposed research. Prof. Dr. Mazliham Mohd Su'ud provided the latest research methodology and research synthesis approaches which helped us to design a novel technique for the prior investigation of flash floods.

\section{REFERENCES}

[1] O. Intharasombat and P. Khoenkaw, "A low-cost flash flood monitoring system," 2015 7th International Conference on Information Technology and Electrical Engineering (ICITEE), Chiang Mai, 2015, pp. 476-479.

[2] K. Achawakorn, K. Raksa and N. Kongkalai, "Flash flood warning system using SCADA system: Laboratory level," 2014 International Electrical Engineering Congress (iEECON), Chonburi, 2014, pp. 1-4.

[3] P. Rapant et al., "Early warning of flash floods based on the weather radar," Proceedings of the 2015 16th International Carpathian Control Conference (ICCC), Szilvasvarad, 2015, pp. 426-430.

[4] S. Muthukumar, W. S. Marry, S. Ajithkumar, M. Arivumathi and V. Sowndharya, "Network based flash flood alert system," 2018 Conference on Emerging Devices and Smart Systems (ICEDSS), Tiruchengode, 2018, pp. 239-241. 
[5] T. Khan, K. Kadir, M. Alcm, Z. Fchiihid and M. S. Mazliham, "Geomagnetic field measurement at earth surface: Flash flood forecasting using tesla meter," 2017 International Conference on Engineering Technology and Technopreneurship (ICE2T), Kuala Lumpur, 2017, pp. 1-4.

[6] F. A. Ruslan, A. M. Samad and R. Adnan, "Modelling of flood prediction system using hybrid NNARX and Extended Kalman Filter," 2017 IEEE 13th International Colloquium on Signal Processing $\&$ its Applications (CSPA), Batu Ferringhi, 2017, pp. 149-152.

[7] T. A. Khan, M. Alam, K. Kadir, Z. Shahid and S. M Mazliham, "A Novel Approach for the Investigation of Flash Floods using Soil Flux and CO2: An Implementation of MLP with Less False Alarm Rate," 2018 2nd International Conference on Smart Sensors and Application (ICSSA), Kuching, 2018, pp. 130-134.

[8] M. Hijji, S. Amin, R. Iqbal and W. Harrop, "A Critical Evaluation of the Rational Need for an IT Management System for Flash Flood Events in Jeddah, Saudi Arabia," 2013 Sixth International Conference on Developments in eSystems Engineering, Abu Dhabi, 2013, pp. 209-214.

[9] M. Mousa, X. Zhang and C. Claudel, "Flash Flood Detection in Urban Cities Using Ultrasonic and Infrared Sensors," in IEEE Sensors Journal, vol. 16, no. 19, pp. 7204-7216, Oct.1, 2016. doi:10.1109/JSEN.2016. 2592359.

[10] T. A. Khan, M. Alam, Z. Shahid and M. M. Suud, "Prior investigation for flash floods and hurricanes, concise capsulization of hydrological technologies and instrumentation: A survey," 2017 IEEE 3rd International Conference on Engineering Technologies and Social Sciences (ICETSS), Bangkok, 2017, pp. 1-6.

[11] N. Bhardwaj, N. Aggarwal, N. Ahlawat and C. Rana, "Controls and intelligence behind "NISTARA-2"-A disaster management machine (DMM)," 2014 Innovative Applications of Computational Intelligence on Power, Energy and Controls with their impact on Humanity (CIPECH), Ghaziabad, 2014, pp. 34-37.

[12] Kotsiantis S.B., Tsekouras G.E., Pintelas P.E. (2005) Bagging Model Trees for Classification Problems. In: Bozanis P., Houstis E.N. (eds) Advances in Informatics. PCI 2005. Lecture Notes in Computer Science, vol 3746. Springer, Berlin, Heidelberg.
[13] L. S. Solanki, S. Singh, and D. Singh, "An ANN approach for false alarm detection in microwave breast cancer detection," in 2016 IEEE Congress on Evolutionary Computation (CEC), 2016, pp. 1370-1374.

[14] O Daniell, Y Petillot, and S Reed., "Unsupervised seafloor classification for automatic target recognition". Proc. International Conf. Remote Sens., (October), 2012.

[15] DP Williams and E Fakiris. Exploiting environmental information for improved underwater target classification in sonar imagery. IEEE Trans. Geosci. Remote Sens., 52(10):6284-6297, 2013.

[16] C. E. Rasmussen, Evaluation of Gaussian processes and other methods for non-linear regression, University of Toronto, 1999.

[17] Suresh, P. V. S., Venkateswara Rao, P. and Deshmukh, S. G., "A Genetic Algorithmic Approach for Optimization of Surface Roughness Prediction Model," International Journal of Machine Tools and Manufacture, Vol. 42, 2002, pp. 675-680.

[18] Yujun Yang, Jianping Li and Yimei Yang, "The research of the fast SVM classifier method," 2015 12th International Computer Conference on Wavelet Active Media Technology and Information Processing (ICCWAMTIP), Chengdu, 2015, pp. 121-124.

[19] C. E. Rasmussen, C. K. Williams, Gaussian processes for machine learning, Vol. 1, MIT press Cambridge, 2006.

[20] Talha Khan, Muhammad Alam, Faraz Shaikh, Sheroz Khan, Kushsairy Kadir, Zeeshan Shahid, M.S Mazliham \& Yahya," Flash floods prediction using real time data: An implementation of ANN-PSO with less false alarm", 2019 IEEE International Instrumentation \& Measurement Technology Conference 20-23 May 2019, Grand Millenium Auckland, New Zealand.

[21] T. Khan, M. Alam, and M. Mazliham, "Artificial Intelligence Based Multi-modal Sensing for Flash Flood Investigation", jictra, pp. 40-47, Jun. 2018.

[22] F. Mercier, N. Akrour, L. Barthès, C. Mallet and R. Hallali, "Fine-scale evaluation of rainfall from TV-sats: A new method for water monitoring and flash flood prevention," in URSI Radio Science Bulletin, vol. 2017, no. 360, pp. 80-88, March 2017. 